\title{
Prediction of Breathing and Gate-Opening Transitions Upon Binary Mixture Adsorption in Metal-Organic Frameworks
}

\author{
François-Xavier Coudert, ${ }^{, \dagger}$ Caroline Mellot-Draznieks, ${ }^{\ddagger}$ Alain H. Fuchs, ${ }^{\dagger}$ and Anne Boutin ${ }^{\S}$ \\ Chimie ParisTech, CNRS and Université Pierre et Marie Curie, 11 rue Curie, F-75005 Paris, France, Department \\ of Chemistry, University College London, 20 Gordon Street, London, WC1H OAJ, United Kingdom, and Laboratoire \\ de Chimie Physique, CNRS and Université Paris-Sud, Bât. 349, F-91405 Orsay Cedex, France
}

Received May 27, 2009; E-mail: fx.coudert@enscp.fr

Among the numerous applications of metal-organic frameworks (MOFs), a topical class of nanoporous materials, adsorptive separation is gaining considerable attention. Some of the most exciting candidates for gas separation processes exhibit structural transitions, such as breathing and gate opening. While predictive analytical methods are crucial in separation science and have been widely used for rigid nanoporous solids, a lack exists for materials that exhibit flexibility. We propose here a general method predicting, for the first time, the evolution of structural transitions and selectivity upon adsorption of gas mixtures in flexible nanoporous solids.

Porous metal-organic frameworks (MOFs) display an extremely large range of crystal structures. The combination of tunable porosity and chemistry of the internal surface opens the way to an extremely rich host-guest chemistry. Many applications have been proposed for MOF materials, including gas storage, catalysis, and sensing. In particular, adsorptive gas separation has seen a rapidly growing interest from the community. Indeed, compared to other classes of microporous materials currently used in gas separation processes (e.g., zeolites, activated carbons, and silica gels), MOFs show a great potential due to the wide possibilities of pre- or postsynthetic functionalization of the organic linker. For a very extensive review of gas adsorption and separation in MOFs, see ref 1 .

A particularly topical class of MOFs are the materials exhibiting a flexible (or dynamic) porous framework, which respond to pressure, temperature, or adsorption of guest molecules by changes in their structure. These systems have received a lot of focus and include materials displaying such eye-catching phenomena as gate opening $^{2,3}$ (transition from a closed, nonmicroporous phase to an open, porous phase), breathing ${ }^{4}$ (two successive structural transitions) upon gas adsorption, and swelling with solvent adsorption. ${ }^{5}$ These systems have been mainly studied from a structural point of view, and their behavior upon adsorption of various guests has been characterized, while simulations of their guest-induced response have only recently been reported. ${ }^{6}$

While many authors have stated that flexible MOFs are good candidates for gas separation, it is to be noted that such predictions are usually based solely on looking at their pure-component adsorption isotherms. An example of direct measurements of adsorption selectivities for a gas mixture in flexible MOFs was provided recently by Denayer et al. on $\mathrm{CO}_{2} / \mathrm{CH}_{4}$ separation in materials of the MIL-53 family, by means of breakthrough experiments. ${ }^{7,8}$ Because the parameter space for studies of binary or ternary mixture coadsorption in nanoporous solids is much bigger

\footnotetext{
${ }^{\dagger}$ Chimie ParisTech, CNRS and Université Pierre et Marie Curie.

* University College London.

$\S$ CNRS and Université Paris-Sud.
}

than that for pure-component adsorption, and especially so in materials that present multiple metastable structures, there is a strong need for a robust theoretical model to help guide the exploration of a large number of materials and working conditions (temperature, pressure, composition) to predict efficient candidates for adsorptive gas separation. In particular, there is currently no information in the literature regarding how guest-induced structural transitions (such as breathing and gate opening) evolve under adsorption of gas mixtures. Indeed, structural transition pressures observed upon pure-component adsorption usually depend strongly on the nature of the adsorbate. ${ }^{3}$ This raises the question of how the structural transition depends on the mixture composition? The most intriguing example of this open question relates to cases where one adsorbate induces a structural transition, while a second adsorbate does not: what happens upon adsorption of a mixture of the two fluids?

We propose here a simple, analytical method to study the evolution of selectivity and host transitions based on experimental pure-component isotherms, which are readily available in the literature. We apply this method to study the evolution of gate opening in $\mathrm{Cu}\left(4,4^{\prime} \text {-bipy)(dhbc }\right)_{2}$ and the breathing of MIL-53 upon adsorption of $\mathrm{CO}_{2} / \mathrm{CH}_{4}$ mixtures, which compares favorably with the experimental data.

To describe the adsorption of a fluid in a flexible porous material, the appropriate thermodynamic ensemble is the osmotic ensemble. For materials exhibiting abrupt structural transitions between different metastable framework structures (as opposed to swelling where progressive structural transformations occur), we demonstrated in earlier work that the use of an "osmotic subensemble" adequately describes the equilibrium between host structures upon adsorption, ${ }^{9}$ rationalizing complex phenomena in flexible MOFs in terms of a competition between the free energy of the material and adsorption energies. ${ }^{10}$ Similarly to the equations for purecomponent adsorption, the thermodynamic potential $\Omega_{\mathrm{os}}^{(k)}$ of a given phase $k$ of the material upon mixture adsorption can be written as

$$
\Omega_{\mathrm{os}}^{(k)}=F_{\mathrm{host}}^{(k)}+P V_{k}-R T \int_{0}^{P} \frac{N_{\mathrm{tot}}^{(k)}(T, p, \mathbf{x})}{p} \mathrm{~d} p
$$

where $N_{\text {tot }}^{(k)}$ is the total quantity of adsorbed fluid, $F_{\text {host }}^{(k)}$ is the free energy of the empty structure, and $V_{k}$ is the unit cell volume of phase $k$.

For each value of composition and pressure of the fluid mixture, the most stable phase is simply the one that has the lowest osmotic potential, allowing us to determine the thermodynamic stability domains for each phase in a pressure-composition diagram. Since host free energies can be calculated from pure-component stepped isotherms, all that is needed for a full determination of the structural transitions upon mixture adsorption is the total quantity of adsorbed fluid $N_{\mathrm{tot}}^{(k)}(P, \mathbf{x})$. This can be determined in a number of ways, 


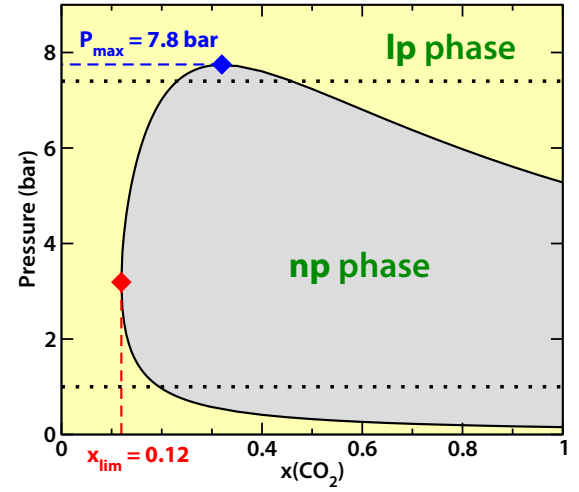

Figure 1. Predicted diagram of stability of the $\mathbf{n p}$ and $\mathbf{l p}$ phases of MIL53 (Al) upon adsorption of a $\mathrm{CO}_{2} / \mathrm{CH}_{4}$ mixture at $304 \mathrm{~K}$, as a function of total pressure and mixture composition. The horizontal dotted lines correspond to the constant-pressure experiments. ${ }^{7}$

including Grand Canonical Monte Carlo molecular simulations, ${ }^{11}$ analytical theories such as the Ideal Adsorbed Solution Theory (IAST), ${ }^{12}$ or other nonideal theories. IAST is expected to yield reasonable results for certain classes of fluids, including small gas molecules and mixtures of apolar fluids of a similar chemical nature (e.g., mixtures of hydrocarbons). The applicability of IAST to $\mathrm{CO}_{2} /$ $\mathrm{CH}_{4}$ separation in MOFs has indeed been validated in recent work by Snurr et al., by comparison with GCMC simulations of the adsorbed mixtures. ${ }^{13}$ The method we propose here consists of combining the osmotic ensemble framework (eq 1) with IAST to predict the evolution of structural transitions upon adsorption of gas mixtures. Our theory is thus based exclusively on purecomponent adsorption isotherms. We call this method the Osmotic Framework Adsorbed Solution Theory, or OFAST.

In this paper, we first highlight the predictive power of the OFAST method on the adsorption of the $\mathrm{CO}_{2} / \mathrm{CH}_{4}$ mixture in metal-organic framework MIL-53 (Al) recently measured by Denayer et al. ${ }^{7}$ The MIL-53 framework topology ${ }^{14}$ is formed of $1 \mathrm{D}$ chains of corner-sharing $\mathrm{MO}_{4}(\mathrm{OH})_{2}$ octahedra $\left(\mathrm{M}=\mathrm{Al}^{3+}, \mathrm{Cr}^{3+}\right)$ linked by 1,4-benzenedicarboxylate (BDC) ligands, which results in linear lozenge-shaped channels large enough to accommodate small guest molecules. This structure may oscillate between two distinct states, a large-pore form (lp) and a narrow-pore form (np; see Figure 1S), depending on gas adsorption and desorption or temperature. This is called the breathing effect. There is up to a $40 \%$ difference in unit cell volume between these two forms. The list of guests that induce this breathing includes $\mathrm{CO}_{2}, \mathrm{H}_{2} \mathrm{O}$, and $\mathrm{C}_{n} \mathrm{H}_{2 n+2}(n \geq 2)$, but neither $\mathrm{H}_{2}$ nor $\mathrm{CH}_{4}$ at room temperature. ${ }^{4,15,16}$ We answer here the question of what happens for a binary mixture of two components, one of which induces breathing while the other does not (e.g., $\mathrm{CO}_{2}$ and $\mathrm{CH}_{4}$, respectively).

In previous work, we have demonstrated that the thermodynamic equations of the osmotic ensemble provide a very good description of the guest-induced breathing upon adsorption of pure $\mathrm{CO}_{2}, \mathrm{CH}_{4}$, or linear alkanes. ${ }^{10,17}$ We used Langmuir fits ${ }^{18}$ of the experimental isotherms as approximations to the "rigid host" isotherms in both lp and np structures; the Langmuir parameters used are summarized in Table 1S. We also calculated the free energy difference between the two structures observed upon $\mathrm{CO}_{2}$ adsorption to be $\Delta F \simeq 2.5$ $\mathrm{kJ} / \mathrm{mol}$ at $304 \mathrm{~K}$ for MIL-53 (Al). ${ }^{9}$ These parameters, all deduced from pure-component adsorption experimental data, are all that is needed in OFAST to predict the coadsorption behavior.

By solving the OFAST equations numerically, we determine for each composition of the $\mathrm{CO}_{2} / \mathrm{CH}_{4}$ mixture whether breathing occurs and what are the two transition pressures, $P_{1}=P_{\mathrm{lp} \rightarrow \mathrm{np}}$ and $P_{2}=$

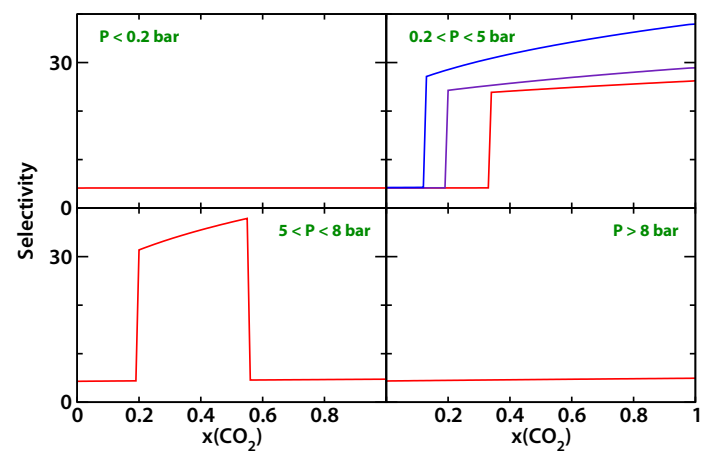

Figure 2. Curves of selectivity as a function of $\mathrm{CO}_{2}$ composition of the mixture, for different fixed values of the total pressure $(0.1,0.5,1,4,7$, and $10 \mathrm{bar}$ ), in MIL-53 (Al) at $304 \mathrm{~K}$.

$P_{\text {np } \rightarrow \text { lp }}$. These two branches, $P_{1}\left(x_{\mathrm{CO}_{2}}\right)$ and $P_{2}\left(x_{\mathrm{CO}_{2}}\right)$, are shown in Figure 1 and demarcate the existence domains of the lp and $\mathbf{n p}$ forms of MIL-53 (Al) in the $\left(x_{\mathrm{CO}_{2}}, P\right)$ phase diagram of the mixture adsorption. The same diagram is clearly visible in the contour plot of $\mathrm{CO}_{2}$ vs $\mathrm{CH}_{4}$ selectivity as a function of pressure and composition, shown in Figure 2S, where the $\mathbf{n p}$ phase can be seen as a highselectivity island (with values of selectivity in the range of a few tens), separated from the lower-selectivity background that is the Ip phase. By looking at vertical lines on this diagram (i.e., considering the adsorption of a mixture of a given composition), we first see that mixture-induced breathing only occurs at a $\mathrm{CO}_{2}$ molar fraction larger than a critical composition of $x_{\lim }\left(\mathrm{CO}_{2}\right) \simeq$ 0.12. For smaller molar fractions of $\mathrm{CO}_{2}$, the mixture behaves like pure $\mathrm{CH}_{4}$ and does not induce breathing, while, for larger $\mathrm{CO}_{2}$ fractions, the two successive structural transitions will take place.

$P_{1}\left(x_{\mathrm{CO}_{2}}\right)$ evolves in a monotonic fashion between $\sim 0.2$ bar (for pure $\mathrm{CO}_{2}$ ) and $\sim 4$ bar (for $x_{\text {lim }}$ ). This monotonic behavior is a generic feature that can be extracted from the low-pressure limit of OFAST. This feature can thus be expected for other mixtures in bistable frameworks such as MIL-53. The behavior of $P_{2}\left(x_{\mathrm{CO}_{2}}\right)$, on the other hand, is not monotonic and goes from 5 bar (for pure $\mathrm{CO}_{2}$ ) to 4 bar (for $x_{\mathrm{lim}}$ ) by reaching a maximum value close to 8 bar, at $x\left(\mathrm{CO}_{2}\right) \simeq 0.3$. ${ }^{19}$ This evolution of $P_{\mathrm{np} \rightarrow \mathrm{p}}$ is quite unexpected and, to our knowledge, has been neither observed experimentally nor hypothesized.

The existence of this maximum for $P_{2}$ is linked with the negative slope of $P_{2}\left(x_{\mathrm{CO}_{2}}\right)$ for $x_{\mathrm{CO}_{2}}$ close to 1 . The sign of the slope is itself due to the evolution of the total adsorbed quantities $N_{\text {tot }}$ in both phases, when a small amount of $\mathrm{CH}_{4}$ is introduced. Upon introduction of $\mathrm{CH}_{4}$, the diminution of the adsorbed quantity $\left(\partial N_{\mathrm{tot}} / \partial x_{\mathrm{CO}_{2}}\right)$ is larger in the lp structure than in the np structure. It is purely an effect of adsorbed quantities, the term $\left(-\mu_{\mathrm{CO}_{2}} N_{\mathrm{CO}_{2}}-\mu_{\mathrm{CH}_{4}} N_{\mathrm{CH}_{4}}\right)$ being dominant at large pressure. We have indeed verified that the total quantity of adsorbed fluid in the np structure upon reopening is almost constant, $N_{\text {tot }}(\mathbf{n p}) \simeq 2.5$ in the range $x\left(\mathrm{CO}_{2}\right) \in[0.35 ; 1]$.

Finally, we can look at the behavior of mixture adsorption as a function of composition, for a fixed value of the total pressure (horizontal lines on Figure 1). The profiles of selectivity and adsorbed quantities along these lines are plotted, respectively, in Figures 2 and 3S. We can distinguish three types of behavior: (i) no structural transition for pressures below 0.2 bar or above 8 bar (lp structure for all compositions); (ii) one transition (lp $\rightarrow \mathbf{n p}$ ) between 0.2 and 5 bar; (iii) two transitions $(\mathbf{l p} \rightarrow \mathbf{n p}$ followed by $\mathbf{n p} \rightarrow \mathbf{l p}$ ) for pressures between 5 and 8 bar.

The picture for adsorbed quantities is more complicated than that, because, in the case of a single $\mathbf{l p} \rightarrow \mathbf{n p}$ transition, it can happen at constant total adsorbed quantity $N_{\text {tot }}$ (if $P=P\left(x_{\text {lim }}\right)$ ) or lead to a 


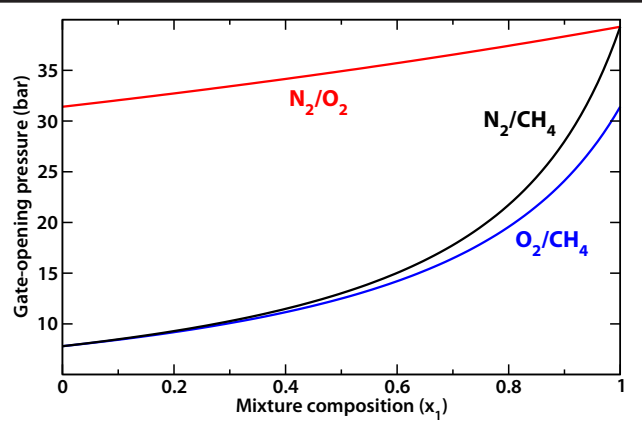

Figure 3. Predicted gate-opening pressure in $\mathrm{Cu}\left(4,4^{\prime}-\right.$ bipy $)(\mathrm{dhbc})_{2}$ at 298 $\mathrm{K}$ for binary mixtures of $\mathrm{N}_{2}, \mathrm{O}_{2}$, and $\mathrm{CH}_{4}$, as a function of mixture composition.

jump in total adsorbed quantity (positive if $P<P\left(x_{\text {lim }}\right)$, negative if $\left.P>P\left(x_{\text {lim }}\right)\right)$. These constant-pressure profiles of MIL-53 (Al) adsorption as a function of varying mixture composition are good tools to help interpret the results of the breakthrough experiments with variable gas composition. ${ }^{7}$ The first thing to note is that the experimental results only contain points for mixture composition above the critical $\mathrm{CO}_{2}$ molar fraction. While, for these compositions, the breathing of MIL-53 is indeed observed as we expect, this does not allow a direct test of the validity of our prediction for $x_{\lim }\left(\mathrm{CO}_{2}\right)$. A second noteworthy point is that the values of selectivity measured in the fixed-bed experiments using MIL-53 pellets with a polyvinyl alcohol binder is an order of magnitude smaller than the results that one obtains from IAST (or from comparing adsorption affinities at low pressure, for that matter). This may be attributed to kinetic effects which are not taken into account by ideal adsorption theories. However, simple IAST is known to overestimate selectivity, and better quantitative agreement would certainly be obtained using a nonideal theory, such as HIAST or an equation of state-based models. Despite that, the $\left(x_{\mathrm{CO}_{2}}, P\right)$ phase diagram that we propose can help explain the behavior of both the experimental selectivity and adsorbed quantities as a function of $x\left(\mathrm{CO}_{2}\right)$ at $7.4 \mathrm{bar}$, which are nonmonotonic. While the authors of ref 7 cannot explain the drop in selectivity as $x\left(\mathrm{CO}_{2}\right)$ increases because they believe that MIL-53 is necessarily in the lp phase for $P>5$ bar, we have shown here that this is not the case and that the selectivity drop can be explained by the $\mathbf{n p} \rightarrow \mathbf{l p}$ transition. This also explains the nonmonotonic evolution of adsorbed quantities, as can be seen in Figure 3S for pressures between 5 and 8 bar (second panel from the top). Both these results underline the existence of an $\mathbf{n p} \rightarrow \mathbf{l p}$ transition at a higher pressure than in the case of pure $\mathrm{CO}_{2}$, an unexpected finding that we predict for the first time using our thermodynamic framework.

OFAST can help rationalize experimental results. We also make two predictions about this system: first, that there is a critical composition $x_{\lim }\left(\mathrm{CO}_{2}\right) \simeq 0.12$ above which breathing occurs for the mixture; second, that the pressure of the second transition $\left(P_{\mathbf{n p} \rightarrow \mathbf{p}}\right)$ does not vary monotonically with the mixture composition and that for some $\mathrm{CO}_{2} / \mathrm{CH}_{4}$ mixtures the transition can happen at higher pressure than that for pure $\mathrm{CO}_{2}$.

As a second application of OFAST, we have studied the gate opening of $\mathrm{Cu}\left(4,4^{\prime} \text {-bipy)(dhbc }\right)_{2}$ upon adsorption of mixtures of $\mathrm{N}_{2}, \mathrm{O}_{2}$, and $\mathrm{CH}_{4}$, for which experimental pure-component adsorption isotherms are available. ${ }^{3}$ We show in Figure 3 the evolution of the gate-opening pressure for the three binary mixtures of these gases, and it can be seen that it varies smoothly and monotonically between the gate-opening pressures for the respective pure components. While no experimental data are available to date on this material to check the validity of our model, the predictions established here appear clear enough that they can be tested experimentally. This is particularly true of the $\mathrm{O}_{2} / \mathrm{CH}_{4}$ and $\mathrm{N}_{2} / \mathrm{CH}_{4}$ mixtures, where the range of evolution of gate-opening pressure is large enough (between 7 and 40 bar) to be analyzable. It is to be noted that our model only deals with the thermodynamic stability of the phases of the material at equilibrium and yields no insight into the hystereses that are systematically observed experimentally.

In conclusion, we proposed here a method for the prediction of structural transitions upon adsorption of gas mixtures in flexible nanoporous metal-organic frameworks, based on experimental pure-component adsorption isotherms, with the aim of guiding the difficult search for optimal materials and operating conditions for gas separation. We showed how this method, which we call OFAST (Osmotic Framework Adsorbed Solution Theory), may anticipate the evolution of breathing and gate-opening processes upon mixture adsorption, in a way that is consistent with the experimental data available to date.

Acknowledgment. We wish to thank G. Baron, J. Denayer, and V. Finsy for fruitful discussions.

Supporting Information Available: Structures of materials studied, selectivity diagram, adsorption isotherms as a function of pressure and composition, analysis of sensitivity, and summary table of the parameters used. This material is available free of charge via the Internet at http://pubs.acs.org.

\section{References}

(1) Li, J.-R.; Kuppler, R. J.; Zhou, H.-C. Chem. Soc. Rev. 2009, 38, $1477-$ 1504.

(2) Tanaka, D.; Nakagawa, K.; Higuchi, M.; Horike, S.; Kubota, Y.; Kobayashi, T. C.; Takata, M.; Kitagawa, S. Angew. Chem., Int. Ed. 2008, 47, 39143918.

(3) Li, D.; Kaneko, K. Chem. Phys. Lett. 2001, 335, 50-56. Kitaura, R.; Seki, K.; Akiyama, G.; Kitagawa, S. Angew. Chem., Int. Ed. 2003, 42, 428-431.

(4) Bourrelly, S.; Llewellyn, P. L.; Serre, C.; Millange, F.; Loiseau, T.; Férey, G. J. Am. Chem. Soc. 2005, 127, 13519-13521.

(5) Serre, C.; Mellot-Draznieks, C.; Surblé, S.; Audebrand, N.; Filinkchuck, Y.; Férey, G. Science 2007, 315, 1828. Mellot-Draznieks, C.; Serre, C.; Surblé, S.; Audebrant, N.; Férey, G. J. Am. Chem. Soc. 2005, 127, 16273.

(6) Coombes, D. S.; Cora, F.; Mellot-Draznieks, C.; Bell, R. G. J. Phys. Chem. C 2009, 113, 544. Salles, F.; Ghoufi, A.; Maurin, G.; Bell, R. G.; MellotDraznieks, C.; Férey, G. Angew. Chem., Int. Ed. 2008, 47, 8487.

(7) Finsy, V.; Ma, L.; Alaerts, L.; De Vos, D. E.; Baron, G. V.; Denayer, J. F. M. Microporous Mesoporous Mater. 2009, 120, 221-227.

(8) Couck, S.; Denayer, J. F. M.; Baron, G. V.; Rémy, T.; Gascon, J.; Kapteijn, F. J. Am. Chem. Soc. 2009, 131, 6326-6327.

(9) Jeffroy, M.; Fuchs, A. H.; Boutin, A. Chem. Commun. 2008, 3275-3277.

(10) Coudert, F.-X.; Jeffroy, M.; Fuchs, A. H.; Boutin, A.; Mellot-Draznieks, C. J. Am. Chem. Soc. 2008, 130, 14294-14302.

(11) Fuchs, A. H.; Cheetham, A. K. J. Phys. Chem. B 2001, 105, 7375-7383.

(12) Myers, A. L.; Prausnitz, J. M. AIChE J. 1965, 11, 121.

(13) Bae, Y.-S.; Mulfort, K. L.; Frost, H.; Ryan, P.; Punnathanam, S.; Broadbelt, L. J.; Hupp, J. T.; Snurr, R. Q. Langmuir 2008, 24, 8592-8598.

(14) Millange, F.; Serre, C.; Férey, G. Chem. Commun. 2002, 822. Serre, C.; Millange, F.; Thouvenot, C.; Nogues, M.; Marsolier, G.; Louër, D.; Férey, G. J. Am. Chem. Soc. 2002, 124, 13519. Liu, Y.; Her, J.-H.; Dailly, A.; Ramirez-Cuesta, A. J.; Neumann, D. A.; Brown, C. M. J. Am. Chem. Soc. 2008, 130, 11813 .

(15) Férey, G.; Latroche, M.; Serre, C.; Millange, F.; Loiseau, T.; PercheronGuegan, A. Chem. Commun. 2003, 2976-2977.

(16) Alaerts, L.; Maes, M.; Giebeler, L.; Jacobs, P. A.; Martens, J. A.; Denayer, J. F. M.; Kirschhock, C. E. A.; De Vos, D. E. J. Am. Chem. Soc. 2008, $130,14170-14178$.

(17) Coudert, F.-X.; Mellot-Draznieks, C.; Fuchs, A. H.; Boutin, A. J. Am. Chem. Soc. 2009, 131, 3442-3443.

(18) The model presented here is not tied to Langmuir fits but could be used with any analytical expression for the isotherms. In the present case, the experimental isotherms are well fitted by the Langmuir equation.

(19) While the exact value of the maximum reached for $P_{2}$ is sensitive to the uncertainties of the parameters used to describe $\mathrm{CH}_{4}$ adsorption in the $\mathbf{n p}$ structure, we have confirmed the nonmonotonic behavior of $P_{2}\left(x_{\mathrm{CO}_{2}}\right)$ and, thus, the existence of the maximum itself, well beyond the range of uncertainty in the parameters (see Figure $5 \mathrm{~S}$ and caption for more details).

JA904123F 